\title{
Detection of associations with rare and common SNPs for quantitative traits: a nonparametric Bayes-based approach
}

\author{
Lili Ding ${ }^{1,2^{*}}$, Tesfaye M Baye ${ }^{2,3}$, Hua He ${ }^{4}$, Xue Zhang ${ }^{4}$, Brad G Kurowski ${ }^{2,5}$, Lisa J Martin ${ }^{1,2,4}$
}

From Genetic Analysis Workshop 17

Boston, MA, USA. 13-16 October 2010

\begin{abstract}
We propose a nonparametric Bayes-based clustering algorithm to detect associations with rare and common single-nucleotide polymorphisms (SNPs) for quantitative traits. Unlike current methods, our approach identifies associations with rare genetic variants at the variant level, not the gene level. In this method, we use a Dirichlet process prior for the distribution of SNP-specific regression coefficients, conduct hierarchical clustering with a distance measure derived from posterior pairwise probabilities of two SNPs having the same regression coefficient, and explore data-driven approaches to select the number of clusters. SNPs falling inside the largest cluster have relatively low or close to zero estimates of regression coefficients and are considered not associated with the trait. SNPs falling outside the largest cluster have relatively high estimates of regression coefficients and are considered potential risk variants. Using the data from the Genetic Analysis Workshop 17, we successfully detected associations with both rare and common SNPs for a quantitative trait. We conclude that our method provides a novel and broadly applicable strategy for obtaining association results with a reasonably low proportion of false discovery and that it can be routinely used in resequencing studies.
\end{abstract}

\section{Background}

The two highly debated hypotheses on the genetic basis of complex human diseases are the common disease/ common variant (CDCV) hypothesis and the common disease/rare variant (CDRV) hypothesis [1]. The CDCV hypothesis states that common diseases are caused by common variants (minor allele frequencies $[\mathrm{MAF}]>5 \%$ ) with small to modest effects. The CDRV hypothesis, on the other hand, argues that common diseases are caused by multiple rare variants (MAF $<5 \%$ ), each with moderate to high penetrance. Although both common and rare variants likely play a role in complex human diseases, most statistical strategies for association analysis have been developed under the CDCV assumption, except recent work by Li and Leal [2] and Han and Pan [3]. A key strategy for association analysis with rare variants is

\footnotetext{
* Correspondence: lili.ding@cchmc.org

'Division of Biostatistics and Epidemiology, Cincinnati Children's Hospital

Medical Center, 3333 Burnet Avenue, Cincinnati, OH 45229, USA

Full list of author information is available at the end of the article
}

to study the cumulative effect of multiple rare variants within the same gene or linkage disequilibrium block $[2,4,5]$. However, these methods identify genetic risk factors at the gene level, not the variant level. We propose a nonparametric Bayes-based approach to detect associations with both rare and common genetic variants for quantitative traits. This approach clusters singlenucleotide polymorphisms (SNPs) according to the magnitude of SNP-specific regression coefficients. SNPs clustered together could come from different linkage disequilibrium blocks, genes, or even different chromosomes and could have quite different MAFs.

\section{Methods}

Suppose that for each individual $i(i=1,2, \ldots, n)$ we observe $y_{i}$, a quantitative trait; $z_{i}$, a $p$-dimensional vector of individual-specific covariates, such as age and sex; and $x_{i}=\left(x_{i 1}, x_{i 2}, \ldots, x_{i J}\right)$, genotypes at $J$ SNPs. Here, we assume an additive genetic model; thus $x_{i j}=0,1$, or 2 , representing the number of minor alleles present at SNP 
$j$ of individual $i$. A regression model on the quantitative trait is given by:

$$
y_{i}=z_{i}^{\prime} \gamma+\sum_{j=1}^{J} x_{i j} \beta_{j}+\varepsilon_{i}, \quad \varepsilon_{i} \sim N\left(0, \sigma^{2}\right),
$$

for $i=1,2, \ldots, n$, where $\gamma$ is a vector of regression coefficients, including the intercept and slopes for individual-specific covariates, the $\beta_{j}$ are the SNP-specific regression coefficients, and $\varepsilon_{i}$ is the error term. We specify the following prior distributions for the model parameters: $\gamma \sim N_{p+1}\left(\mathbf{0}, \mathbf{1}, v^{2}\right), \beta_{j} \sim G, G \sim \mathrm{DP}\left(\alpha, G_{0}\right)$, and $\sigma \sim U(a, b)$. Here, $v^{2}$ and $b>a \geq 0$ are prespecified hyperparameters, $N_{p+1}(\boldsymbol{\mu}, \Sigma)$ is a $(p+1)$-dimensional normal distribution with mean vector $\boldsymbol{\mu}$ and variancecovariance matrix $\Sigma, \mathbf{0}$ is a vector of zeros, $\mathbf{I}$ is an identity matrix, $G$ denotes a random distribution, $U(a, b)$ denotes a uniform distribution between $a$ and $b$, and DP $\left(\alpha, G_{0}\right)$ is the Dirichlet process.

\section{Dirichlet process}

The Dirichlet process [6] is a probability model on a space of probability distributions. It has two parameters: the base probability distribution $G_{0}$ and the precision parameter $\alpha(>0)$. If $G \sim \operatorname{DP}\left(\alpha, G_{0}\right)$, then $G_{0}$ is the prior expectation of $G$ and $\alpha$ controls the variance of $G$. Here, we take $G_{0}=N+\left(\mu_{0}, \sigma_{0}^{2}\right)$, which is a normal density truncated below at 0 , and use $U(c, d), d>c>0$, as the prior distribution for the precision parameter $\alpha$.

Sethuraman [7] provided a stick-breaking construction of the Dirichlet process, which states that if we have:

$$
\begin{aligned}
& p_{1}=V_{1}, \\
& p_{k}=V_{k} \prod_{j=1}^{k-1}\left(1-V_{j}\right), \quad k=2,3, \ldots, \\
& V_{k} \sim \operatorname{Beta}(1, \alpha),
\end{aligned}
$$

and

$$
\phi_{k} \sim G_{0}, k=1,2, \ldots,
$$

then

$$
G=\sum_{k=1}^{\infty} p_{k} \delta_{\phi_{k}}
$$

is a random probability distribution generated from $\operatorname{DP}\left(\alpha, G_{0}\right)$, where $\delta_{\varphi_{k}}$ denotes a point mass at $\varphi_{k}$. It is clear that $G$ is discrete with probability 1 . Because of the discreteness, the $\beta_{j}$ can take on the same value. That is why the Dirichlet process can be used for clustering analysis.

Ishwaran and James [8] studied a truncated version of the Dirichlet process by choosing a truncation level $N$ and setting $V_{N}=1$ in the stick-breaking construction. They used the truncated Dirichlet process to approximate Dirichlet process prior distributions and developed a block Gibbs sampling method for Dirichlet process models.

\section{Clustering}

Each iteration of the Gibbs sampler gives a clustering structure of SNP-specific regression coefficients such that coefficients taking the same value are clustered together. The number of clusters and the cluster membership of the coefficients vary across iterations, giving a random sample of clustering structures. Pairwise probabilities of two coefficients being equal are calculated from the posterior samples [9]. A distance measure is derived as 1 minus these pairwise probabilities and is then used in complete linkage hierarchical clustering to obtain a final clustering structure of the SNPs. We study a range of the number of clusters, from as small as 2-5 clusters to as large as 100 clusters. Optimal cluster numbers are also obtained by striking a balance between sensitivity and specificity. In all cases, SNPs in the largest cluster have relatively low or close to zero estimates of regression coefficients and are considered not associated with the trait. SNPs falling outside the largest cluster have relatively high estimates of regression coefficients and are considered potential risk variants. The proportion of false discovery (FDP), defined as the ratio of the number of false discoveries to the total number of discoveries, is examined.

\section{Application of the method}

We illustrate our methods using the data from Genetic Analysis Workshop 17. The analyses were performed with the knowledge of the underlying simulation model [10]. We studied the first 10 replicates of the quantitative trait Q1. Each replicate contains 697 unrelated individuals from 7 populations. To control for population stratification, we conducted principal components analysis on nonsynonymous common SNPs $(n=1,379)$ and included the resulting first two components as covariates, in addition to Age and Smoke. We built our model with 244 nonsynonymous SNPs selected from the vascular endothelial growth factor (VEGF) pathway [11]. These SNPs include all 39 functional SNPs for Q1, of which 23 are private variants (found in one individual, MAF $=0.000717)$ and 2 are common SNPs. The model was fitted using WinBUGS [12] with $v^{2}=1,000, a=0$, $b=100, c=0.5, d=20, \mu_{0}=0.5$, and $\sigma_{0}^{2}=2$. The truncation level for the Dirichlet process was fixed at 
Table 1 True discoveries in at least two replicates with 2 to 5 clusters

\begin{tabular}{lccccccc}
\hline Gene & SNP & MAF & $\boldsymbol{\beta}$ & F2 & F3 & F4 & F5 \\
\hline$F L T 1$ & C13S523 & 0.066714 & 0.64997 & $\mathbf{1 0}$ & $\mathbf{1 0}$ & $\mathbf{1 0}$ & $\mathbf{1 0}$ \\
$F L T 1$ & $C 13 S 431$ & 0.017217 & 0.74136 & $\mathbf{9}$ & $\mathbf{9}$ & $\mathbf{9}$ & $\mathbf{9}$ \\
$F L T 1$ & $C 13 S 522$ & 0.027977 & 0.61830 & $\mathbf{8}$ & $\mathbf{8}$ & $\mathbf{8}$ & $\mathbf{8}$ \\
VEGFA & C6S2981 & 0.002152 & 1.20645 & $\mathbf{6}$ & $\mathbf{6}$ & $\mathbf{6}$ & $\mathbf{7}$ \\
$A R N T$ & $C 1 S 6533$ & 0.011478 & 0.5619 & $\mathbf{5}$ & $\mathbf{6}$ & $\mathbf{7}$ & $\mathbf{7}$ \\
$F L T 1$ & $C 13 S 524$ & 0.004304 & 0.62223 & $\mathbf{4}$ & $\mathbf{4}$ & $\mathbf{5}$ & $\mathbf{5}$ \\
$K D R$ & $C 4 S 1884$ & 0.020803 & 0.29558 & $\mathbf{4}$ & $\mathbf{4}$ & $\mathbf{4}$ & $\mathbf{5}$ \\
$K D R$ & $C 4 S 1878$ & 0.164993 & 0.13573 & $\mathbf{2}$ & $\mathbf{4}$ & $\mathbf{4}$ & $\mathbf{4}$ \\
$K D R$ & $C 4 S 1877$ & 0.000717 & 1.07706 & 1 & $\mathbf{4}$ & $\mathbf{5}$ & $\mathbf{6}$ \\
$K D R$ & $C 4 S 1889$ & 0.000717 & 0.94133 & 1 & $\mathbf{2}$ & $\mathbf{3}$ & $\mathbf{5}$ \\
$A R N T$ & $C 1 S 6542$ & 0.002152 & 0.46026 & 1 & $\mathbf{2}$ & $\mathbf{2}$ & $\mathbf{2}$ \\
$K D R$ & $C 4 S 1861$ & 0.002152 & 0.56311 & 1 & 1 & 1 & $\mathbf{2}$ \\
\hline
\end{tabular}

$F 2, F 3, F 4$, F5: frequency of detection (in bold when $\geq 2$ ) over the 10 replicates with 2 to 5 clusters.

50. For each replicate, 10,000 Markov chain Monte Carlo posterior samples were generated after a burn-in period of 2,000 iterations.

We evaluated our results using two thresholds. When the number of clusters was small (2-5), we defined true positives as true associations identified in at least 2 of the 10 replicates. This threshold was selected to balance the reduced power resulting from small cluster numbers. Indeed, requiring at least two replications for each identified association yielded a reasonably low FDP. When we used the optimal cluster numbers, we defined true positives as true associations detected in no less than eight replicates. We carried out sensitivity analyses on the prior specification for the SNP-specific regression coefficients with $\mu_{0}$ ranging from 0.1 to 0.5 and $\sigma_{0}^{2}$ ranging from 0.5 to 2 . Similar results were obtained.

\section{Results and discussion \\ Successful identification of associations}

Table 1 lists the true discoveries with their MAFs, regression coefficients $(\beta)$ used in the simulation, and frequency of detection. When we used two clusters, we found eight true positives with no false positives. Compared with false negatives, the true positives have either relatively high MAFs or relatively high effect sizes. With 3 clusters, we had 11 true positives and no false positives. With 4 or 5 clusters, we detected 12 and 13 associations, respectively. However, there was one false positive (data not shown) in both cases (FDP $\approx 8 \%$ ). We also conducted single-SNP-based tests with Bonferroni correction for multiple comparisons. With the criteria that $p \leq 0.05 / 244$ in at least 2 of the 10 replicates, 20 associations were detected and 10 of them were true discoveries, giving an FDP of $50 \%$. Compared with single-SNP tests, our method gave a much lower FDP.

\section{Selection of optimal number of clusters}

As the number of clusters increases, more associations may be detected; however, the number of false positives may also increase. To strike a balance between sensitivity and specificity, we examined receiver operating characteristic (ROC) curves (Figure 1) for each replicate. The optimal cluster numbers ranged from 59 to 96 , with an

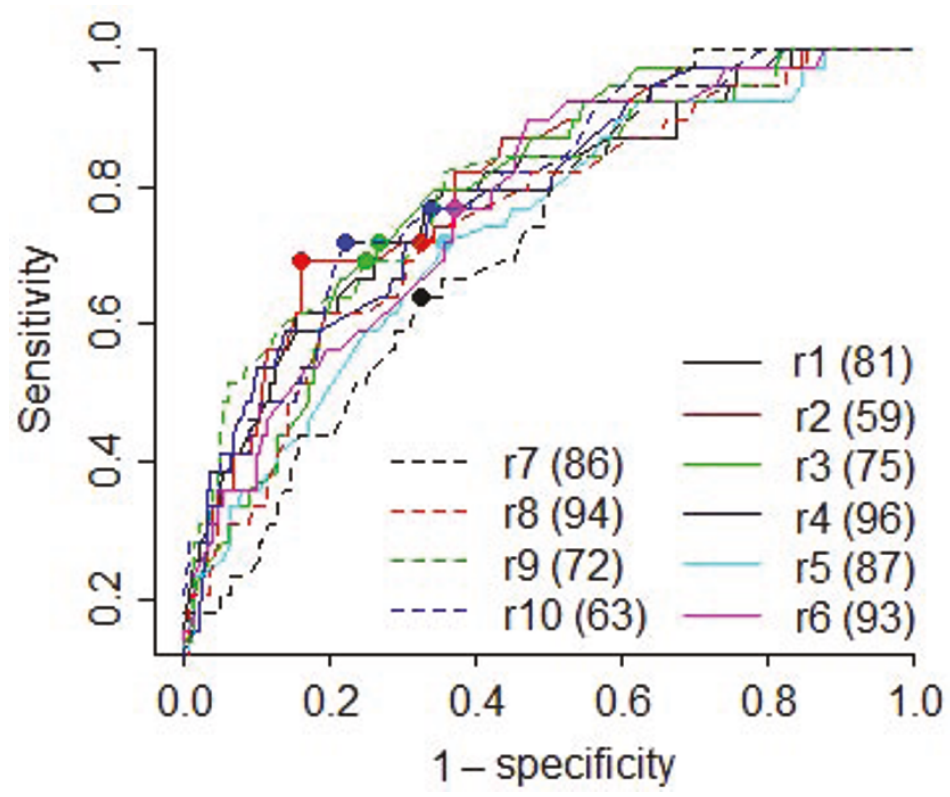

Figure 1 ROC curves and optimal cluster numbers. $r_{1}-r_{10}$ represent the first 10 replicates of the quantitative trait Q1. Numbers in parentheses and dots on the curves indicate optimal number of clusters for each replicate, which ranges from 59 to 96, with an average of 81. 
Table 2 True discoveries in at least eight replicates with optimal cluster numbers

\begin{tabular}{|c|c|c|c|}
\hline Gene & SNP & MAF & $\beta$ \\
\hline \multicolumn{4}{|c|}{$F=10, \mathrm{TP}=10, \mathrm{FP}=2, \mathrm{FDP}=17 \%$} \\
\hline ARNT & C1S6561 & 0.000717 & 0.65721 \\
\hline$K D R$ & C4S1877 & 0.000717 & 1.07706 \\
\hline$K D R$ & C4S1879 & 0.000717 & 0.61830 \\
\hline$K D R$ & C4S1889 & 0.000717 & 0.94133 \\
\hline VEGFC & C4S4935 & 0.000717 & 1.35726 \\
\hline VEGFA & C6S2981 & 0.002152 & 1.20645 \\
\hline FLT1 & C13S431 & 0.017217 & 0.74136 \\
\hline FLT1 & C13S522 & 0.027977 & 0.61830 \\
\hline FLT1 & C13S523 & 0.066714 & 0.64997 \\
\hline FLT1 & C13S524 & 0.004304 & 0.62223 \\
\hline \multicolumn{4}{|c|}{$F=9, \mathrm{TP}=15, \mathrm{FP}=2, \mathrm{FDP}=12 \%$} \\
\hline ELAVL4 & C1S3181 & 0.000717 & 0.76911 \\
\hline ELAVL4 & C1S3182 & 0.000717 & 0.30432 \\
\hline ARNT & C1S6533 & 0.011478 & 0.56190 \\
\hline FLT1 & C13S399 & 0.000717 & 0.39602 \\
\hline FLT1 & C13S479 & 0.000717 & 0.75946 \\
\hline \multicolumn{4}{|c|}{$F=8, \mathrm{TP}=19, \mathrm{FP}=4, \mathrm{FDP}=17 \%$} \\
\hline$K D R$ & C4S1873 & 0.000717 & 0.58301 \\
\hline$K D R$ & C4S1884 & 0.020803 & 0.29558 \\
\hline FLT4 & C5S5156 & 0.000717 & 0.43010 \\
\hline FLT1 & C13S505 & 0.000717 & 0.44850 \\
\hline
\end{tabular}

The items in the bolded rows are the frequency of detection over the 10 replicates (F), the number of true positives (TP), the number of false positives (FP), and the proportion of false discovery (FDP) in each case.

average of 81 . At the optimal cluster number for each replicate, the average sensitivity and specificity were 0.71 and 0.72 , respectively. We then examined the associations detected in these rounds. We had $100 \%$ power (detected in all 10 replicates) to detect 10 true associations (Table 2) with 2 false positives (FDP $=17 \%)$. Using a threshold of $90 \%$ power, five additional true associations were detected and are in the $F=9$ rows in Table 2, with no additional false positives (FDP $=12 \%)$. Using a threshold of $80 \%$ power, we detected another four true associations (19 total); however, the number of false positives went up to four (FDP $=17 \%$ ).
We then evaluated the performance of this method using a specified number of clusters, ranging from 50 to 100 . Using only associations identified with $100 \%$ power, we had 8 to 10 true positives and at most 2 false negatives (FDP ranging from $10 \%$ to $18 \%$ ). For $90 \%$ power, we had 8 to 16 true positives and at most 4 false negatives (FDP ranging from $8 \%$ to $20 \%$ ). Thus cluster numbers of 50 to 100 seem reasonable.

Characteristics of the true positives and false negatives Using optimal cluster numbers and the threshold of true positives, we identified 12 of the 23 true associations with
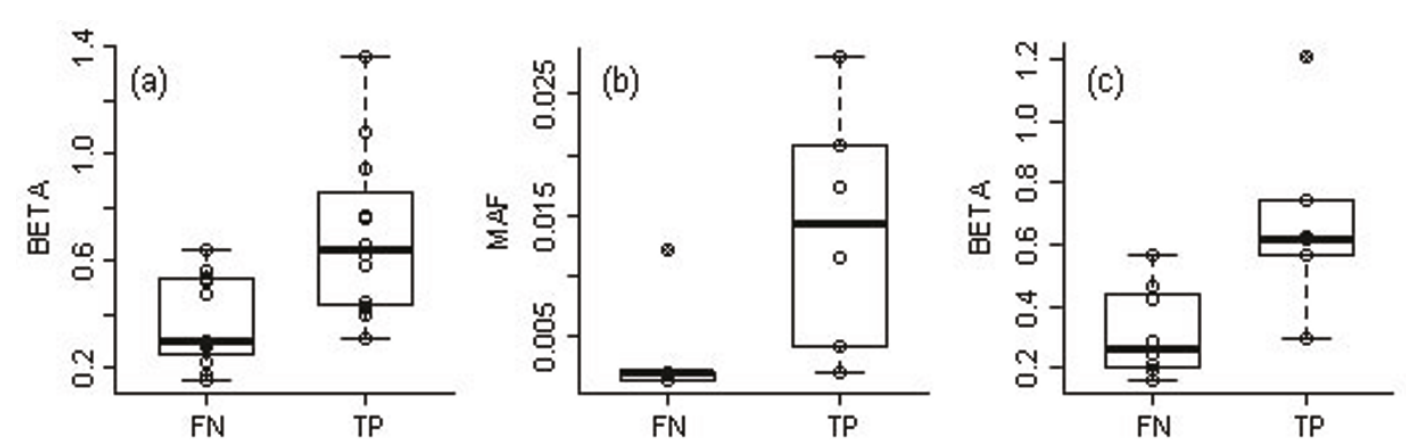

Figure 2 Boxplot of $\beta$ and MAF by false-negative or true-positive status. (a) $\beta$ of private functional SNPs (MAF $=0.000717$ ) for Q1; (b) MAF and (c) $\beta$ of rare but nonprivate functional SNPs $(0.000717<$ MAF $<0.05)$ for Q1. FN, false negative; TP, true positive. 
private SNPs. As we expected, true positives had overall higher effect sizes than false negatives (Figure 2a). Among the 14 true associations with rare but nonprivate variants, true positives had relatively high MAFs and $\beta$ compared with false negatives, as shown in Figures $2 \mathrm{~b}$ and $2 \mathrm{c}$.

\section{Conclusions}

We have demonstrated that a novel nonparametric Bayesbased clustering method can be used to identify associations with SNPs for quantitative traits. Importantly, this method is capable of detecting associations with both rare and common genetic variants. Compared with other methods that deal with rare variants, our methods detect genetic risk factors directly at the SNP level. Compared with single-SNP-based methods, the proposed method is more powerful and reliable. It can detect a relatively larger proportion of true associations independent of the MAF of the variants, and it produces a relatively lower proportion of false discoveries.

\section{Acknowledgments}

We wish to thank Siva Sivaganesan for valuable discussion and two anonymous reviewers for their insightful comments and suggestions. This work was supported by National Institutes of Health $(\mathrm{NIH})$ grants $\mathrm{K} 01$ HL103165, K12 HD001097-14, K24 HL69712, R01 NS036695, and U19 A1070235. The Genetic Analysis Workshops are supported by NIH grant R01 GM031575 from the National Institute of General Medical Sciences. This article has been published as part of BMC Proceedings Volume 5 Supplement 9, 2011: Genetic Analysis Workshop 17. The full contents of the supplement are available online at http://www.biomedcentral.com/1753$6561 / 5$ ? issue $=\$ 9$.

\section{Author details}

'Division of Biostatistics and Epidemiology, Cincinnati Children's Hospital Medical Center, 3333 Burnet Avenue, Cincinnati, OH 45229, USA. 2Department of Pediatrics, University of Cincinnati College of Medicine, Cincinnati OH 45229, USA. ${ }^{3}$ Division of Asthma Research, Cincinnati Children's Hospital Medical Center, 3333 Burnet Avenue, Cincinnati, $\mathrm{OH}$ 45229, USA. ${ }^{4}$ Division of Human Genetics, Cincinnati Children's Hospital Medical Center, 3333 Burnet Avenue, Cincinnati, OH 45229, USA. ${ }^{5}$ Division of Physical Medicine and Rehabilitation, Cincinnati Children's Hospital Medical Center, 3333 Burnet Avenue, Cincinnati, OH 45229, USA.

\section{Authors' contributions}

LD conceived and performed the statistical analysis and wrote the manuscript. LMM contributed to the design of the statistical analysis and the writing of the manuscript. TMB, HH, XZ, and BGK helped with the writing of the manuscript. All authors read and approved the final manuscript.

\section{Competing interests}

The authors declare that there are no competing interests.

Published: 29 November 2011

\section{References}

1. Schork NJ, Murray SS, Frazer KA, Topol EJ: Common vs. rare allele hypotheses for complex diseases. Curr Opin Genet Dev 2009, 19:212-219.

2. Li B, Leal SM: Methods for detecting associations with rare variants for common diseases: application to analysis of sequence data. Am J Hum Genet 2008, 83:311-321.

3. Han F, Pan W: A data-adaptive sum test for disease association with multiple common or rare variants. Hum Hered 2010, 70:42-54.
4. Morgenthaler S, Thilly WG: A strategy to discover genes that carry multiallelic or mono-allelic risk for common diseases: a cohort allelic sums test (CAST). Mutat Res Fund Mol Mech 2007, 615:28-56.

5. Madsen BE, Browning SR: A groupwise association test for rare mutations using a weighted sum statistic. PLoS Genet 2009, 5:e1000384.

6. Ferguson TS: Bayesian analysis of some nonparametric problems. Ann Stat 1973, 1:209-230

7. Sethuraman J: A constructive definition of Dirichlet priors. Stat Sinica 1994, 4:639-650.

8. Ishwaran H, James LF: Gibbs sampling methods for stick-breaking priors. J Am Stat Assoc 2001, 96:161-173.

9. Medvedovic M, Sivaganesan S: Bayesian infinite mixture model based clustering of gene expression profiles. Bioinformatics 2002, 18:1194-1206.

10. Almasy LA, Dyer TD, Peralta JM, Kent JW Jr, Charlesworth JC, Curran JE, Blangero J: Genetic Analysis Workshop 17 mini-exome simulation. BMC Proc 2011, 5(suppl 9):S2.

11. BioCarta: Pathways: VEGF, hypoxia, angiogenesis.[http://www.biocarta. com/pathfiles/h_vegfpathway.asp].

12. Lunn DJ, Thomas A, Best N, Spiegelhalter D: WinBUGS: a Bayesian modeling framework-concepts, structure, and extensibility. Stat Comput 2000, 10:325-337.

doi:10.1186/1753-6561-5-S9-S10

Cite this article as: Ding et al:. Detection of associations with rare and common SNPs for quantitative traits: a nonparametric Bayes-based approach. BMC Proceedings 2011 5(Suppl 9):S10.

\section{Submit your next manuscript to BioMed Central and take full advantage of:}

- Convenient online submission

- Thorough peer review

- No space constraints or color figure charges

- Immediate publication on acceptance

- Inclusion in PubMed, CAS, Scopus and Google Scholar

- Research which is freely available for redistribution 